\title{
Evaluation of the Effect of Aminophylline in Reducing the Incidence of Acute Kidney Injury After Cardiac Surgery
}

\author{
Shahrbanoo Shahbazi, ${ }^{1}$ Peyman Alishahi, ${ }^{1}$ and Elham Asadpour ${ }^{1,}$ \\ ${ }^{1}$ Anesthesiology and Critical Care Research Center, Shiraz University of Medical Sciences, Shiraz, Iran \\ "Corresponding author: Elham Asadpour, Anesthesiology and Critical Care Research Center, Shiraz University of Medical Sciences, Nemazee Hospital, Shiraz, Iran. Tel/Fax: \\ +98-7136281460, E-mail: asadpoure@sums.ac.ir
}

Received 2017 April 17; Revised 2017 June 14; Accepted 2017 August 07.

\begin{abstract}
Objectives: Acute renal failure is a common complication of major cardiovascular surgeries (One-third of patients). Adenosine release as a vascular vasodilator increases after cardiac surgery, which reduces renal and glomerular blood flow and subsequently causes kidney ischemic damage. The present study aimed at evaluating the impact of aminophylline as an adenosine receptor antagonist on renal function after cardiac surgery hoping to find an appropriate method to reduce acute kidney injury.

Methods: The patients in the intervention group received $5 \mathrm{mg} / \mathrm{kg}$ aminophylline bolus after induction of anesthesia; then, 0.25 $\mathrm{mg} / \mathrm{kg} / \mathrm{hr}$ of the drug was administered intraoperatively and up to 48 hours after surgery in the ICU cardiac surgery. Similar volume of normal saline was injected to the patients of the second group. Serum BUN, Cr, and GFR were measured pre- and postoperatively and 3 days postsurgery. Patients' 24- hour urine output and RIFLE were also calculated.

Results: Those patients who received medication were extubated earlier $(\mathrm{P}=0.018)$ and received lower amount of inotropic drugs $(\mathrm{P}<0001)$. According to the RIFLE criteria, most of the patients experienced no change or even improved GFR and $\mathrm{Cr}$ amounts compared to the control group $(\mathrm{p}<0.05)$. GFR and $\mathrm{Cr}$ value of all the patients with Cleveland score, less and more than 6 , showed a significant difference between the 2 groups $(\mathrm{P}=0.001$ and $\mathrm{P}=0.01$, respectively). According to the RIFLE criteria, most of the patients experienced no change or even improved GFR.

Conclusions: Aminophylline in cardiac surgery can reduce the frequency of acute kidney injury according to RIFLE criteria and could be used in the prevention of AKI as a safe and efficient modality in high-risk patients. Also, the use of this drug may reduce the need for inotropic medication at the time of surgery, intensive care unit stay length, and extubation time.
\end{abstract}

Keywords: Aminophylline, Acute Kidney Injury, Cardiac Surgery, Glomerular Filtration Rate, Creatinine

\section{Background}

Acute renal failure based on its definition, sudden worsening of kidney function and reduced glomerular filtration $(1,2)$, is a common complication of important diseases, major surgeries, and sepsis (3). Acute kidney injury (AKI) is a well-known and prevalent side effect, especially after cardiac surgery, which varies from asymptomatic rises in biochemical markers up to dialysis necessity (4-11).

In cardiac surgery, acute kidney injury may be caused by several mechanisms. During cardiac surgery, especially in cases of cardiovascular instability, usually the mean arterial pressure is less than $80 \mathrm{mmHg}$. Also, many of these patients have impaired system of kidney self-regulation to keep the glomerular filtration until the mean arterial pressure less than $80 \mathrm{~mm} \mathrm{Hg}$ is reached, which is due to associated conditions such as aging, atherosclerosis, hypertension, and chronic renal failure, impaired renal function using nephrotoxic drugs, or proinflammatory conditions. Another reason is the initiation of systemic inflam- matory response syndrome (SIRS). In fact, proinflammatory conditions such as damage caused by surgery, blood contact with artificial surfaces during cardiopulmonary bypass (CPB), and endotoxemia play a key role in AKI development via inflammation. It seems that SIRS resulting from cardiac surgery works in the same way (4).

Adenosine release as a vascular vasodilator increases after cardiac surgery, which reduces renal and glomerular blood flow, and subsequently causes kidney ischemic damage (12).

Cleveland model is the most common model to predict the probability of AKI after cardiac surgery. AKI risk factors are as follow: female gender (1 point), congestive heart failure ( 2 points), left ventricular dysfunction(1 point), intraaortic balloon pump before surgery ( 2 points), chronic obstructive pulmonary disease (COPD) (1 point), insulindependent diabetes(1 point), a history of previous cardiac surgery(1 point), emergent cardiac surgery ( 2 points), and heart valve surgery with/without CABG (3 points), other cardiac surgery (2 points), preoperative creatinine (Cr) between 1.2 to 2.1 (2 points), and preoperative $\mathrm{Cr}$ greater than 
2.1 (5 points); the sum of these points show the Cleveland score (Cleveland clinic foundation acute renal failure scoring system) $(1,13)$.

According to Cleveland model, each of the invoices takes certain points, which ranges between 0 and 17 as a whole. All the patients were categorized based on 3 groups of low with a value of 0 to 2, intermediate with 3 to 5 , and high with a value of 6 or larger (14). Accordingly, individuals, who had a higher score, were at a higher risk of AKI. Age, primary disease of the kidney, renal artery disease, hypertension, and atherosclerosis have been identified in other studies as risk factors $(1,3,13,15)$.

RIFLE criteria (risk (increased $\mathrm{Cr} \times 1.5$ or GFR decreases $>25 \%$, or $\mathrm{UO}<0.5 \mathrm{~mL} / \mathrm{kg} / \mathrm{hr} \times 6 \mathrm{hrs}$ ), injury (increased $\mathrm{Cr}$ $\times 2$ or GFR decreases $>50 \%$, or $\mathrm{UO}<0.5 \mathrm{~mL} / \mathrm{kg} / \mathrm{hr} \times 12 \mathrm{hrs}$ ), failure (increased $\mathrm{Cr} \times 3$ or GFR decreases $>75 \%$, or $\mathrm{Cr} \geq 4$ $\mathrm{mg} / \mathrm{dL}$, with acute rise of $\geq 0.5 \mathrm{mg} / \mathrm{dL}$, or $\mathrm{UO}<0.3 \mathrm{~mL} / \mathrm{kg} / \mathrm{hr}$ $\times 24$ hrs, or anuria $\times 12$ hrs), loss of kidney function (persistent ARF = complete loss of renal function $>4$ weeks), and end-stage renal disease (ESRD)) are used to evaluate the postoperative kidney injury $(1,4,14,16)$.

Treatment with aminophylline, as an antagonist of nonspecific adenosine receptor, increases renal filtration and urine flow and reduces the dialysis necessity (12). Gaffney et al. found that AKI could not significantly be reduced after cardiac surgery (6). In another study, aminophylline showed no effect to prevent acute kidney injury in children recovering from cardiac surgery performed with cardiopulmonary bypass (17). The association between perioperative use of aminophylline infusion and lower incidence of deterioration in renal function following cardiac surgery in high-risk patients has been suggested by Mahaldar et al. (14).

The present study aimed at evaluating the impact of aminophylline on renal function after cardiac surgery hoping to find an appropriate method to reduce AKI.

\section{Methods}

This was a double- blind randomized controlled trial that evaluated the effect of aminophylline on renal function after cardiac surgery. This study conformed to the ethical guidelines of the 2000 Declaration of Helsinki as reflected in a priori approval by the Iranian Registry of Clinical Trials (IRCT No.: 2015010619470N10),. The sample size was calculated as 144 patients based on the obtained results of similar studies with the $\mathrm{P} 1=0.14, \mathrm{P} 2=0.015, \alpha=5 \%$, and the power of $80 \%$. Those patients without arrhythmia, tachycardia, nonoliguric kidney injury, anuria, obstructive uropathy, and congenital anomalies were candidates for cardiac surgery.
After receiving approval from the ethics committee of Shiraz University of Medical Sciences, we obtained informed consent from all 144 patients (95 male and 50 female patients). The primary end points were described as glomerular filtration rate (GFR) and $\mathrm{Cr}$ and its relationship with the Cleveland score. Secondary end points were the development of severe acute kidney injury, time between intensive care unit(ICU) admission and first successful extubation, CPB time, blood transfusion volume, urine output(UO), ICU and post intensive care unit (PICU), and length of hospital stay.

In the preoperative examination, the Cleveland score was measured and the patients were categorized based on 3 groups: low, intermediate, and high.

Random numbers were produced from http://www.randomizer.org/ by simple randomization technique, and 2 customized collections of random numbers were generated, which were called 1 and 2 . Based on these 2 sets, the patients were assigned into 2 groups of 72 patients.

Routine care was performed for all patients before and after cardiac surgery. The intervention group received 5 $\mathrm{mg} / \mathrm{kg}$ aminophylline (Sina Daroo, Tehran, Iran) bolus before surgery and after anesthesia induction; then, 0.25 $\mathrm{mg} / \mathrm{kg} / \mathrm{hr}$ of the drug was administered intraoperatively and up to 48 hours after surgery in the ICU cardiac surgery. Similar volume of normal saline was injected to the second group. Serum BUN and Cr, as a marker of kidney function, were measured pre- and postoperatively and for 3 days after the surgery. GFR was measured using the formula of Cockcroft and Gault according to age and serum $\mathrm{Cr}$ (CG GFR $=(140-$ age $) \times$ Weight $/ 72 \times \mathrm{Cr})$; this formula was multiplied by 0.85 for females (14). Patients' 24 - hour UO was calculated up to 2 days after surgery. RIFLE criteria were calculated as well.

Anesthesia was induced by midazolam $0.1-0.2 \mathrm{mg} / \mathrm{kg}$, sufentanil 0.7-1 mcg/kg, sodium thiopental 1-2 mg/kg, and pancuronium $0.2 \mathrm{mg} / \mathrm{kg}$. Anesthesia was maintained using regular inhaled anesthetic agents (eg, isoflurane) during the surgery. All the recruited patients were operated on by one specified surgeon; and the type of inotropic drugs, amount and type of fluids, and blood products used by the surgeon during the operation, aortic cross clamping (ACC), and CPB time were recorded.

In the postoperative ICU, aminophylline was prepared for the infusion by an anesthesia technician for 2 days and placed on infusion pump; and the in charge nurses in the ICU were not aware which patients were receiving the drug or saline. In case of aminophylline medical complications, the ICU supervisor was notified and the drug injection was stopped according to the cardiologist opinion.

The data collected from the study groups were ana- 
lyzed by SPSS 16 software (SPSS Inc., Chicago, IL). Normality analysis was done by Kolmogorov-Smirnov test normality; the obtained quantitative data were analyzed using MannWhitney U test; and the qualitative data analysis was performed by chi-square and t test.

\section{Results}

The mean age of the patients in this study was $61.31 \pm$ 11.40 years. No significant differences were detected between the 2 groups in the demographic data of the patients including age, weight, height, and $\mathrm{CPB}$ time $\left(\mathrm{P}^{*}>0.05\right)$. Based on the Cleveland score, as a risk predictor of AKI after cardiac surgery, patients in each group were divided into 2 groups: patients with a score less than 6 , and patients with a score greater than 6 . Accordingly, among 62 patients in the intervention group (86.1\%) and 63 control participants (87.5\%), with a score less than 6 based on the statistical analysis, the difference was not quite significant $\left(\mathrm{P}^{*}=\right.$ 0.806). similarly, no statistically significant difference was detected between the 2 groups (13.9\% of the patients were in the intervention group and $12.5 \%$ in the control group) for the score of more than $6\left(\mathrm{P}^{*}=0.805\right)$. Moreover, during the operation, 48 patients in the intervention group and 52 in the control group required blood transfusion, and even though fewer individuals in the intervention group received blood transfusion, the difference was not statistically significant. Thus, blood transfusion could not evaluate other outcomes as a confounding factor. Nevertheless, the patients who received medication were extubated earlier than those in the control group, with a significant difference $\left(\mathrm{P}^{*}=0.018\right)$.

Postoperation characteristics such as UO were not significantly different between the 2 groups, but the length of ICU and PICU stay were significantly lower in the treatment group $\left(\mathrm{P}^{*}=0.001\right)$.

During the operation, 51 patients in the intervention group and 65 in the control group have required inotropic medication. Fewer people in the intervention group received inotropic drugs and there was a significant difference between the 2 groups $\left(\mathrm{P}^{*}<0001\right)$ (Error! Reference source not found.).

GFR and Cr. values fluctuation in the treatment group was smaller than the placebo group although the differences were not significant in all the follow-up days except for postoperation GFR in the placebo group, which was significantly lower than the preoperation amount $\left(\mathrm{P}^{*}<0.01\right)$ (Error! Reference source not found.).

In both groups, there was a GFR reduction and $\mathrm{Cr}$ elevation as a result of the increasing duration of $\mathrm{CPB}$ and ACC, and the strength was improved by the length of CPB. In other words, the relationship between reduced GFR and increased $\mathrm{Cr}$ with increasing duration of CPB was weakly significant $\left(\mathrm{P}^{*}=0.056\right.$ and $\mathrm{P}^{*}=0.055$, respectively), but this relationship was not significant based on the ACC time ( $\mathrm{P}^{*}$ $=0.28$ and $\mathrm{P}^{*}=0.18$, respectively).

According to the RIFLE criteria, only 3 patients experienced the GFR reduction of more than 25\% (risk level) in the treatment group, 1 patient experienced 50\% reduction (injury level), and most patients experienced no change or even improved GFR; these amounts were significantly lower than that of the placebo group $\left(\mathrm{P}^{*}<0.05\right)$. Accordingly, $\mathrm{Cr}$ enhancement in the treatment group occurred only in 2 patients up to 1.5 -fold (risk level) and 2 patients up to 2-fold (injury level); and again, the $\mathrm{Cr}$ amounts did not change or even decreased in most of the patients; these values were significantly lower than that of the placebo group $\left(\mathrm{P}^{*}<0.05\right)$. None of the patients in this study were located in the failure, loss, and ESRD groups. Based on GFR data, the frequency of AKI in aminophylline group was 5\% and it was $29.11 \%$ in the control group (Error! Reference source not found.).

GFR value of all the patients with Cleveland score of less and more than 6 was $61.77 \pm 21.97$ and $47.73 \pm 22.25$, respectively, indicating a significant difference between the 2 groups $\left(\mathrm{P}^{*}=0.001\right)$. This amount was $64.35 \pm 20.08$ in aminophylline group by Cleveland score less than 6 , indicating no significant difference compared to the placebo group with the GFR values of $59.23 \pm 23.56\left(P^{*}=0.139\right)$, but GFR value of aminophylline group was $57.06 \pm 19.58$ with Cleveland score greater than 6 , indicating a significant increase compared to that of the placebo group by value of $37.37 \pm 21.29\left(\mathrm{P}^{*}=0.43\right)$ (Error! Reference source not found.).

Similarly, Cr values of all the patients with Cleveland score less than 6 was $1.24 \pm 0.37$, which was significantly lower than that of the group with Cleveland score greater than 6 with the value of $1.65 \pm 0.56\left(\mathrm{P}^{*}=0.01\right)$. Furthermore, comparison between the groups that received aminophylline $(1.21 \pm 0.27)$ and placebo $(1.27 \pm 0.45)$ showed no significant difference in the amount of $\mathrm{Cr}$ in the placebo group when the Cleveland score was less than $6\left(\mathrm{P}^{*}\right.$ $=0.91$ ). However, these amounts were significantly different in the treatment group $(1.31 \pm 0.28)$ and placebo group $(2.03 \pm 0.55)$ with the Cleveland score greater than $6\left(\mathrm{P}^{*}=\right.$ 0.002) (Error! Reference source not found.).

In this study, 3 patients experienced tachycardia, and according to the cardiologist opinion, the treatment drug was stopped for 1 to 2 hours and started again. Only 1 patient from the control group died 4 days after surgery. 
Figure 1. GFR and Cr. Values Fluctuation During the Follow-Up Days

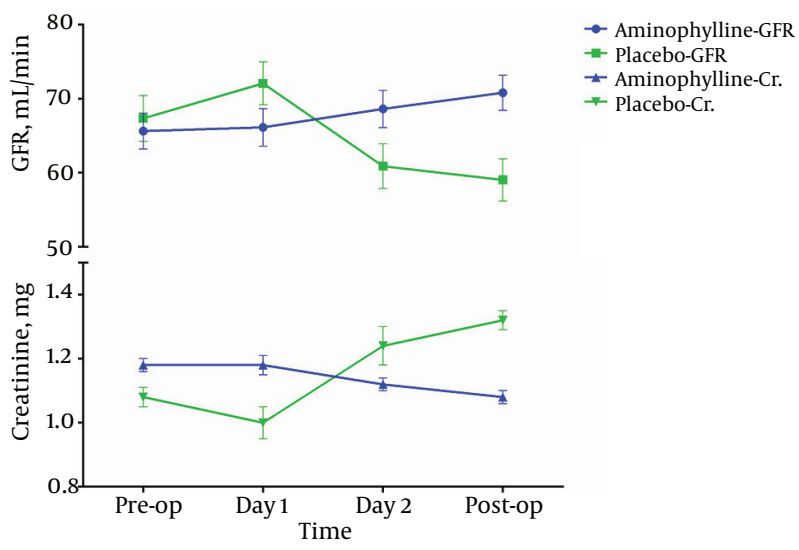

Data are presented as mean \pm SEM.

Figure 2. GFR Values of the Patients in All, Aminophylline and Placebo Groups

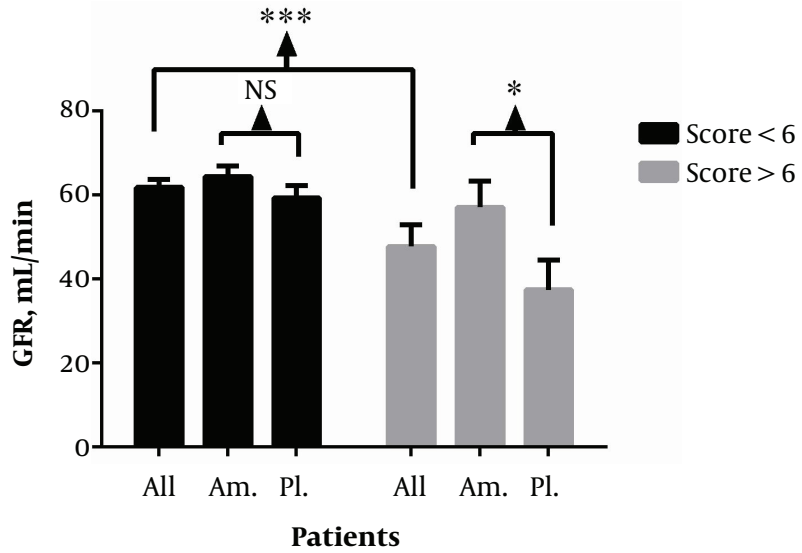

Data are presented as mean \pm SEM, $\left(\mathrm{P}^{*}>0.05\right.$ as NS, ${ }^{*} \mathrm{P}^{*}<0.05$ and $\left.{ }^{* * *} \mathrm{P}^{*}<0.001\right)$.

\section{Discussion}

Considering the mechanism of acute kidney injury after cardiac surgery and the risk factors, this study was designed in 2014-2015 to evaluate the effect of aminophylline to prevent kidney damage. Based on regression analysis, it appears that aminophylline, independent of other confounding factors such as CPB and ACC time, preoperation GFR, and $\mathrm{Cr}$, could reduce postoperative kidney injury according to GFR and Cr criteria.

Our results revealed that in the group that received aminophylline, fewer patients needed the inotropic drug, which could be as a result of positive inotropic effect of aminophylline (3). The extubation time was shorter in the treatment group, which was due to the fact that many fac-
Figure 3. Creatinine Values of the Patients in All, Aminophylline and Placebo Groups

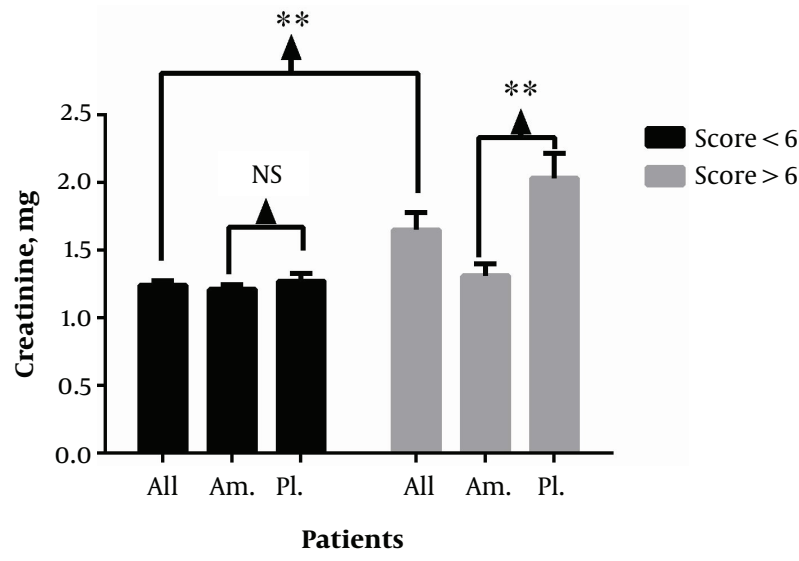

Data are presented as mean $\pm \mathrm{SEM},\left(\mathrm{P}^{*}>0.05\right.$ as NS, $\left.{ }^{* *} \mathrm{P}^{*}<0.01\right)$

Table 1. Baseline and Post Operation Characteristics of the Patients and Operations ${ }^{\mathrm{a}}$

\begin{tabular}{lccc}
\hline Variables & Aminophylline & Placebo & PValue \\
\hline Age, $\mathbf{y}$ & $61.3 \pm 10.09$ & $65.6 \pm 10.10$ & 0.128 \\
\hline Weight, Kg & $66.9 \pm 10.4$ & $65.5 \pm 11.7$ & 0.460 \\
\hline Height, cm & $164.7 \pm 8.21$ & $160.8 \pm 8.92$ & 0.400 \\
\hline Cleveland Score & $3.9 \pm 1.2$ & $3.9 \pm 1.3$ & 0.20 \\
\hline CPB time, min & $83.97 \pm 38.98$ & $71.94 \pm 21.97$ & 0.072 \\
\hline $\begin{array}{l}\text { Blood transfusion } \\
\text { volume, Bag }\end{array}$ & $1.47 \pm 0.61$ & $1.76 \pm 0.87$ & 0.125 \\
\hline Extubation time, hour & $12.65 \pm 10.7$ & $17.11 \pm 11.8$ & 0.018 \\
\hline $\begin{array}{l}\text { First day urine output, } \\
\text { cc/kg/hr }\end{array}$ & $1.45 \pm 0.39$ & $1.46 \pm 0.46$ & 0.098 \\
\hline $\begin{array}{l}\text { Second day urine output, } \\
\text { cc/kg/hr }\end{array}$ & $1.38 \pm 0.39$ & $1.31 \pm 0.45$ & 0.209 \\
\hline ICU stay length, day & $2.08 \pm 0.36$ & $2.63 \pm 1.15$ & 0.001 \\
\hline \begin{tabular}{l} 
PICU stay length, day \\
\hline
\end{tabular} & $4.34 \pm 0.67$ & $4.87 \pm 1.23$ & 0.001 \\
\hline
\end{tabular}

${ }^{\mathrm{a}}$ Data are presented as mean $\pm \mathrm{SD}$.

Table 2. The Number of Patients in the Intervention and the Control Group and Inotropic Drugs During Surgery

\begin{tabular}{lcccc}
\hline Group & Epinephrine & Norepinephrine & Dopamine & Total \\
\hline Aminophylline & 36 & 8 & 7 & 51 \\
Placebo & 63 & 1 & 1 & 65 \\
Total & 99 & 9 & 8 & 116 \\
\hline
\end{tabular}

tors (e.g., anesthetic drugs) could be involved at the time of extubation of the trachea. Thus, this result may not be outstanding for the effect of aminophylline. Similarly, Axelrod 
Table 3. RIFLE Criteria Based on the Results of Postoperative GFR and Cr, Data Are Presented As the Patients' Number

\begin{tabular}{|c|c|c|c|c|c|c|c|c|}
\hline Variables & Group & Total & No Risk & Risk & Injury & Failure & Loss & ESRD \\
\hline \multirow{2}{*}{ GFR } & Aminophylline & 72 & 68 & 3 & 1 & 0 & 0 & 0 \\
\hline & Placebo & 72 & 51 & 18 & 3 & 0 & 0 & 0 \\
\hline \multirow{2}{*}{$\mathbf{C r}$} & Aminophylline & 72 & 69 & 2 & 1 & 0 & 0 & 0 \\
\hline & Placebo & 72 & 53 & 14 & 3 & 0 & 0 & 0 \\
\hline
\end{tabular}

et al. found no significant difference between first successful extubation in the patients who received aminophylline and placebo in preventing acute kidney injury (17).

Rising CPB and ACC time led to increased serum creatinine and reduced GFR in all the patients. It seemed that aminophylline independent of other confounding factors such as CPB and ACC time, preoperation GFR, and creatinine could influence GFR and $\mathrm{Cr}$; this finding is consistent with other studies demonstrating that prolonged time of CPB and cross-clamp affected serum Cr levels and GFR as useful parameters for postoperative renal injury $(18,19)$.

Similar to other studies that used aminophylline or theophylline as adenosine receptor antagonist, we found significant differences between the groups according to RIFLE criteria in assessing the extent of postoperative kidney damage compared with the preoperative (20). As the prominent role of adenosine and its receptors on kidney function and inflammatory processes has been recognized, the potential preventive effect of aminophylline on AKI may be related to its regulatory effects on adenosine receptors (21).

In our study, the patients with the Cleveland score less than 6 showed no significant difference in the GFR and $\mathrm{Cr}$ values compared to the placebo group although the placebo group had worse postoperative renal situation in GFR and creatinine compared to the treatment group. However, comparing the amount of GFR and $\mathrm{Cr}$ in patients with Cleveland score more than 6 in the 2 groups, we found that the average amounts of GFR and Cr were significantly better in the treatment group compared to the control group. The higher amount of preoperative Cleveland score had a noteworthy impact on the postoperative kidney injury in the intervention group, which could be improved by aminophylline. In contrast to the study by Kramer et al. that found no effect of theophylline on preventing AKI in cardiac surgery patients, it was found that aminophylline infusion in high-risk patients with Cleveland score higher than 6 could lead to a better GFR and Crvalues after surgery (22). These results are consistent with the study of Mahaldar et al., which indicated that intravenous aminophylline could be used in the prevention of AKI as a safe and efficient modality among the high-risk patients after cardiac surgery (14).

In our study, aminophylline did not result in improved UO in the intervention group, but the length of stay in the ICU and PICU was lower, which could reduce the health care costs.

In the present study, we did not have access to any drugs as a standard treatment of AKI to compare the results with positive controls. We assessed the response of a single dose of aminophylline without a dose-response titration on the prevention of AKI in patients undergoing cardiac surgery; and finally, a longer follow-up could help gain more consistent results. For the future investigations, other existing pathways responsible for AKI and the therapeutic properties of other agents with similar effects as adenosine receptor antagonists could help find a standard remedy for prophylaxis of AKI. Thus, further studies are warranted in this area.

\subsection{Conclusion}

As a result, it appears that the use of aminophylline before, during, and after heart surgery can reduce the incidence of acute kidney injury. Also, use of this drug may reduce the need for inotropic medication at the time of surgery. The effect of aminophylline on extubation time may not be obvious in patients due to the impact of other factors. However, our study revealed that patients were extubated faster and their length of stay in the intensive care unit was lower compared to the control group. More importantly, aminophylline could reduce the incidence of AKI according to RIFLE criteria and could be used in the prevention of AKI as a safe and efficient modality for the high-risk patients after cardiac surgery.

\section{Acknowledgments}

This article is the result of a thesis written by P. Alishahi MD submitted to the school of medicine in partial fulfillment of the requirements for the degree of specialty in Anesthesiology. 


\section{Footnotes}

Authors' Contribution: Study concept and design, Shahrbanoo Shahbazi, Peyman Alishahi, and Elham Asadpour; analysis and interpretation of data, Peyman Alishahi, and Elham Asadpour; drafting of the manuscript, Elham Asadpour; critical revision of the manuscript for important intellectual content, Shahrbanoo Shahbazi, Peyman Alishahi, and Elham Asadpour; statistical analysis: Shahrbanoo Shahbazi, Peyman Alishahi, and Elham Asadpour.

Financial Disclosure: We have no financial interests related to the material in the manuscript.

Ethics Number: CP-P-9374-7224

Funding/Support: This study was also funded by a grant number 7224 from Vice-Chancellery of research and technology in Shiraz University of Medical Sciences, Shiraz, Iran.

\section{References}

1. Vives M, Wijeysundera D, Marczin N, Monedero P, Rao V. Cardiac surgery-associated acute kidney injury. Interact Cardiovasc Thorac Surg. 2014;18(5):637-45. doi: 10.1093/icvts/ivu014. [PubMed: 24535092].

2. Lynch BA, Gal P, Ransom JL, Carlos RQ, Dimaguila MA, Smith MS, et al. Low-dose aminophylline for the treatment of neonatal non-oliguric renal failure-case series and review of the literature. J Pediatr Pharmacol Ther. 2008;13(2):80-7. doi: 10.5863/1551-6776-13.2.80. [PubMed: 23055869].

3. Parker MR, Willatts SM. A pilot study to investigate the effects of an infusion of aminophylline on renal function following major abdominal surgery. Anaesthesia. 2001;56(7):670-5. doi: 10.1046/j.13652044.2001.01374.x. [PubMed: 11437769].

4. Karkouti K, Wijeysundera DN, Yau TM, Callum JL, Cheng DC, Crowther $\mathrm{M}$, et al. Acute kidney injury after cardiac surgery: focus on modifiable risk factors. Circulation. 2009;119(4):495-502. doi: 10.1161/CIRCULATIONAHA.108.786913. [PubMed: 19153273].

5. Hansen MK, Gammelager H, Jacobsen CJ, Hjortdal VE, Layton JB, Rasmussen BS, et al. Acute Kidney Injury and Long-term Risk of Cardiovascular Events After Cardiac Surgery: A Population-Based Cohort Study. J Cardiothorac Vasc Anesth. 2015;29(3):617-25. doi: 10.1053/j.jvca.2014.08.020. [PubMed: 25575408].

6. Gaffney AM, Sladen RN. Acute kidney injury in cardiac surgery. Curr Opin Anaesthesiol. 2015;28(1):50-9. doi: 10.1097/ACO.0000000000000154. [PubMed: 25486486]

7. Kandler K, Jensen ME, Nilsson JC, Moller CH, Steinbruchel DA. Acute kidney injury is independently associated with higher mortality after cardiac surgery. J Cardiothorac Vasc Anesth. 2014;28(6):1448-52. doi: 10.1053/j.jvca.2014.04.019. [PubMed: 25440657].

8. Berg KS, Stenseth R, Wahba A, Pleym H, Videm V. How can we best predict acute kidney injury following cardiac surgery?: a prospective observational study. Eur J Anaesthesiol. 2013;30(11):704-12. doi: 10.1097/EJA.ob013e328365ae64. [PubMed: 24067536].
9. Dardashti A, Ederoth P, Algotsson L, Bronden B, Bjursten H. Incidence, dynamics, and prognostic value of acute kidney injury for death af ter cardiac surgery. J Thorac Cardiovasc Surg. 2014;147(2):800-7. doi 10.1016/j.jtcvs.2013.07.073. [PubMed: 24100099].

10. Ge Ng RR, Huey Chew ST, Liu W, Kah Ti L. Persistent kidney injury at hospital discharge after cardiac surgery with cardiopulmonary bypass in patients with normal preoperative serum creatinine and normal estimated glomerular filtration rate. J Cardiothorac Vasc Anesth. 2014;28(6):1453-8. doi: 10.1053/j.jvca.2014.05.007. [PubMed: 25263770].

11. Harel Z, Chan CT. Predicting and preventing acute kidney injury after cardiac surgery. Curr Opin Nephrol Hypertens. 2008;17(6):624-8. doi 10.1097/MNH.ob013e32830f4590. [PubMed: 18941357].

12. Olowu WA, Adefehinti O. Aminophylline improves urine flow rates but not survival in childhood oliguric/anuric acute kidney injury Arab J Nephrol Transplant. 2012;5(1):35-9. [PubMed: 22283864].

13. Rosner $\mathrm{MH}$, Okusa MD. Acute kidney injury associated with cardiac surgery. Clin J Am Soc Nephrol. 2006;1(1):19-32. doi: 10.2215/CJN.00240605. [PubMed: 17699187].

14. Mahaldar AR, Sampathkumar K, Raghuram AR, Kumar S, Ramakrishnan M, Mahaldar DA. Risk prediction of acute kidney injury in cardiac surgery and prevention using aminophylline. Indian J Nephrol. 2012;22(3):179-83. doi:10.4103/0971-4065.98752. [PubMed: 23087551].

15. Krzych L, Wybraniec M, Chudek J, Bochenek A. Perioperative management of cardiac surgery patients who are at the risk of acute kidney injury. Anaesthesiol Intensive Ther. 2013;45(3):155-63. doi: 10.5603/AIT.2013.0033. [PubMed: 24092513].

16. Lopez-Delgado JC, Esteve F, Torrado H, Rodriguez-Castro D, Carrio ML, Farrero E, et al. Influence of acute kidney injury on short- and longterm outcomes in patients undergoing cardiac surgery: risk factors and prognostic value of a modified RIFLE classification. Crit Care. 2013;17(6):R293. doi: 10.1186/cc13159. [PubMed: 24330769].

17. Axelrod DM, Sutherland SM, Anglemyer A, Grimm PC, Roth SJ. A Double-Blinded, Randomized, Placebo-Controlled Clinical Trial of Aminophylline to Prevent Acute Kidney Injury in Children Following Congenital Heart Surgery With Cardiopulmonary Bypass. Pediatr Crit Care Med. 2016;17(2):135-43. doi: 10.1097/PCC.0000000000000612. [PubMed: 26669642].

18. Bahar T, Kaplan M, Muruvvet Y, Nehir SU, Tolga C, Adlan O, et al. Cardiovascular Surgery with Cardiopulmonary Bypass in Patients with Preoperative Non-dialysis Dependent Renal Insufficiency. Heart Surg Forum. 2015;18(2):E067-73. doi:10.1532/hsf.1249. [PubMed: 25924035].

19. Luciani R, Lazzarino AI, Capuano F, Benedetto U, Goracci M, Pirozzi N et al. Preoperative creatinine clearance as a predictor of short-term outcomes after cardiac surgery: a cohort study for the comparison between the Cockroft-Gault and modification of diet in renal disease formulae. J Cardiovasc Med (Hagerstown). 2010;11(4):271-5. doi: 10.2459/JCM.ob013e328336b558. [PubMed: 20072000].

20. Kapoor A, Kumar S, Gulati S, Gambhir S, Sethi RS, Sinha N. The role of theophylline in contrast-induced nephropathy: a case-control study Nephrol Dial Transplant. 2002;17(11):1936-41. doi:10.1093/ndt/17.11.1936. [PubMed: 12401850].

21. Vallon V, Osswald $\mathrm{H}$. Adenosine receptors and the kidney. Handb Exp Pharmacol. 2009(193):443-70. doi: 10.1007/978-3-540-89615-9_15. [PubMed: 19639291].

22. Kramer BK, Preuner J, Ebenburger A, Kaiser M, Bergner U, Eilles C, et al. Lack of renoprotective effect of theophylline during aortocoronary bypass surgery. Nephrol Dial Transplant. 2002;17(5):910-5. doi 10.1093/ndt/17.5.910. [PubMed: 11981083]. 